International Journal of Pure and Applied Mathematics

Volume 106 No. 4 2016, 1057-1066

ISSN: 1311-8080 (printed version); ISSN: 1314-3395 (on-line version)

url: http://www.ijpam.eu

doi: 10.12732 /ijpam.v106i4.7

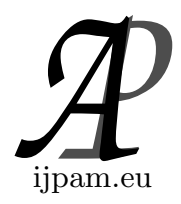

\title{
A NOTE ON EXACT SEQUENCES IN SEMIABELIAN CATEGORIES
}

\author{
Dinamérico P. Pombo Jr. \\ Instituto de Matemática \\ Universidade Federal do Rio de Janeiro \\ Caixa Postal 68530, 21945-970, Rio de Janeiro, RJ BRASIL
}

\begin{abstract}
Necessary and sufficient conditions for the exactness of certain sequences of morphisms in a semiabelian category (in the sense of Palamodov) are established.
\end{abstract}

AMS Subject Classification: 18E05, 18E99, 46M18

Key Words: semiabelian categories, exact sequences, strict morphisms

\section{Introduction}

Two fundamental theorems of Linear Algebra [3, p.227 and p.229] assert that the exactness of certain sequences of linear mappings between modules is equivalent to the exactness of sequences of group homomorphisms between the corresponding abelian groups of linear mappings, both results being valid for arbitrary abelian categories $[2, \mathrm{p} .12 ; 5, \mathrm{p} .128]$. The purpose of this note is to extend these results to the context of semiabelian categories (in the sense of Palamodov). In particular, the results obtained here are applicable to important semiabelian categories occuring in Functional Analysis and Topological Algebra which are not abelian. As we shall see, the notion of a strict morphism will play an important role in our discussion.

Received: October 26, 2015

Published: March 8, 2016
(C) 2016 Academic Publications, Ltd.

url: www.acadpubl.eu 


\section{Preliminaries}

Let $\mathcal{C}$ be a category $[4,5], \mathrm{Ob}(\mathcal{C})$ the class of objects in $\mathcal{C}$ and, for $A, B \in \mathrm{Ob}(\mathcal{C})$, let $\operatorname{Mor}_{\mathcal{C}}(A, B)$ be the set of morphisms from $A$ into $B$; for each $A \in \mathrm{Ob}(\mathcal{C})$ let $1_{A}$ be the identity morphism of $A$.

Let $A \in \mathrm{Ob}(\mathcal{C})$ be fixed and consider all pairs $(L, \ell)$, where $L \in \mathrm{Ob}(\mathcal{C})$ and $\ell: L \rightarrow A$ is an injective morphism in $\mathcal{C}$. If $\left(L^{\prime}, \ell^{\prime}\right)$ and $\left(L^{\prime \prime}, \ell^{\prime \prime}\right)$ are two such pairs, we shall write $\left(L^{\prime}, \ell^{\prime}\right) \leq\left(L^{\prime \prime}, \ell^{\prime \prime}\right)$ if there exists a $w \in \operatorname{Mor}_{\mathcal{C}}\left(L^{\prime}, L^{\prime \prime}\right)$ making the diagram

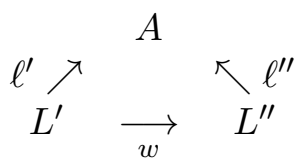

commutative. In this case $w$ is unique and injective. It is obvious that $\leq$ is a preorder on the class of all such pairs $(L, \ell)$. Two pairs $\left(L^{\prime}, \ell^{\prime}\right)$ and $\left(L^{\prime \prime}, \ell^{\prime \prime}\right)$ are said to be equivalent if $\left(L^{\prime}, \ell^{\prime}\right) \leq\left(L^{\prime \prime}, \ell^{\prime \prime}\right)$ and $\left(L^{\prime \prime}, \ell^{\prime \prime}\right) \leq\left(L^{\prime}, \ell^{\prime}\right)$; in this case if we write $\ell^{\prime}=\ell^{\prime} w$ and $\ell^{\prime \prime}=\ell^{\prime} w_{1}$ (as above), then $w$ and $w_{1}$ are isomorphisms and $w^{-1}=w_{1}$. In each class of equivalent pairs we shall choose a pair (for example, by means of Hilbert's tau-symbol), called a subobject of $A$; the class of subobjects of $A$ is an ordered class with respect to $\leq$. Dually, one defines the notion of a quotient of $A$.

A category $\mathcal{C}$ is said to be preadditive [2] if the following axioms hold:

(a) for all $A, B \in \operatorname{Ob}(\mathcal{C})$, $\operatorname{Mor}_{\mathcal{C}}(A, B)$ is endowed with an abelian group structure (the identity element of $\operatorname{Mor}_{\mathcal{C}}(A, B)$ will be denoted by $O_{A B}$ );

(b) the composition of morphisms is a $\mathbb{Z}$-bilinear mapping;

(c) there exists an object $A$ in $\mathcal{C}$ which is a zero of $\mathcal{C}$, that is, $1_{A}=O_{A A}$ (since between two zeros of $\mathcal{C}$ there is only one isomorphism, namely, the only morphism between them, we shall refer to the zero of $\mathcal{C}$, denoted by $O$ ).

Let $\mathcal{C}$ be a preadditive category and $u \in \operatorname{Mor}_{\mathcal{C}}(A, B)$. A pair $(L, \ell)$, where $L \in \mathrm{Ob}(\mathcal{C})$ and $\ell \in \operatorname{Mor}_{\mathcal{C}}(L, A)$, is said to be a generalized kernel of u [5] if, for each $D \in \operatorname{Ob}(\mathcal{C})$, the sequence

$$
O \longrightarrow \operatorname{Mor}_{\mathcal{C}}(D, L) \stackrel{\ell^{*}}{\longrightarrow} \operatorname{Mor}_{\mathcal{C}}(D, A) \stackrel{u^{*}}{\longrightarrow} \operatorname{Mor}_{\mathcal{C}}(D, B)
$$

of group homomorphisms is exact, where $\ell^{*}(\varphi)=\ell \varphi$ for $\varphi \in \operatorname{Mor}_{\mathcal{C}}(D, L)$ and $u^{*}(\psi)=v \psi$ for $\psi \in \operatorname{Mor}_{\mathcal{C}}(D, A)$. For $(L, \ell)$ to be a generalized kernel of $u$ it is necessary and sufficient that $u \ell=O_{L B}, \ell$ be an injective morphism and $\operatorname{Ker}\left(u^{*}\right) \subset \operatorname{Im}\left(\ell^{*}\right)$. Among the generalized kernels of $u$ (if they do exist) there is exactly one which is a subobject of $A$, denoted by $(\operatorname{Ker}(u), i)$ and called the 
kernel of $u$ ( $i$ is said to be the canonical morphism). Dually one defines the cokernel of $u$, denoted by (Coker $(u), j$ ) ( $j$ is said to be the canonical morphism).

Let $\mathcal{C}$ be a preadditive category such that $\operatorname{Ker}(u)$ and $\operatorname{Coker}(u)$ exist for any morphism $u$ in $\mathcal{C}$. For $u \in \operatorname{Mor}_{\mathcal{C}}(A, B)$, the coimage of $u$ is defined by $\operatorname{Coim}(u)=$ Coker $(\operatorname{Ker}(u))$ and the image of $u$ is defined by $\operatorname{Im}(u)=\operatorname{Ker}(\operatorname{Coker}(u))$. If $A \stackrel{\alpha}{\longrightarrow} \operatorname{Coim}(u)$ and $\operatorname{Im}(u) \stackrel{\beta}{\longrightarrow} B$ are the canonical morphisms, there is a unique morphism $\operatorname{Coim}(u) \stackrel{\bar{u}}{\longrightarrow} \operatorname{Im}(u)$ in $\mathcal{C}$ so that $u=\beta \bar{u} \alpha[2$, p.5; 5, p.126].

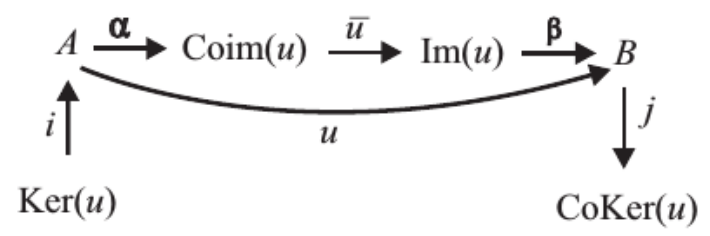

\section{Exact Sequences in Semiabelian Categories}

Definition $3.1[8]$. A category $\mathcal{C}$ is said to be semiabelian if it is preadditive, $\operatorname{Ker}(u)$ and $\operatorname{Coker}(u)$ exist for any morphism $u$ in $\mathcal{C}$, the coproduct $A \sqcup B$ of $A, B$ exists for all $A, B \in \mathrm{Ob}(\mathcal{C})$ (or equivalently, the product $A \times B$ of $A, B$ exists for all $A, B \in \mathrm{Ob}(\mathcal{C})$; in this case $A \sqcup B$ and $A \times B$ are isomorphic [2, p.2; 5, p.126]) and $\bar{u}$ is a bijective morphism for any morphism $u$ in $\mathcal{C}$.

Example 3.2. The following categories are semiabelian, as one may readily verify [10]:

(a) the category of unitary left $R$-modules, where $R$ is a ring with a non-zero identity element [3, Chapter 2] (more precisely, this is an abelian category);

(b) the category of unitary left topological $R$-modules, where $R$ is a topological ring with a non-zero identity element $[13, \S 12]$;

(c) the category of unitary left linearly topologized $R$-modules, where $R$ is a topological ring with a non-zero identity element [13, §31];

(d) the category of abelian Hausdorff topological groups [13, Chapter I];

(e) the category of (real or complex) Banach spaces [7, 11];

(f) the category of (real or complex) locally convex spaces [7, 11];

(g) the category of locally convex spaces over a complete non-Archimedean non-trivially valued field [12]; 
(h) the category of bornological $R$-modules, where $R$ is a topological ring with a non-zero identity element [9];

(i) the category of (real or complex) convex bornological vector spaces [6];

(j) the category of $K$-convex bornological vector spaces over a complete non-Archimedean non-trivially valued field $K$ [1].

For the rest of this note $\mathcal{C}$ will denote an arbitrary semiabelian category, unless otherwise specified.

Definition 3.3. A morphism $u$ in $\mathcal{C}$ is said to be strict if the corresponding morphism $\bar{u}$ is an isomorphism.

Example 3.4. If $A \stackrel{u}{\longrightarrow} B$ is an arbitrary morphism in $\mathcal{C}$ and $(L, \ell)$ is a generalized kernel of $u$, then $\ell$ is a strict morphism.

First let us recall that, for a morphism $T \stackrel{t}{\longrightarrow} A$ in $\mathcal{C}$, we have that $t=O_{T A}$ if and only if $\operatorname{Coker}(t)=\left(A, 1_{A}\right)$. Thus, since $\ell$ is injective, $\operatorname{Ker}(\ell)=\left(O, O_{O L}\right)$ by Proposition 4 of [2], and hence

$$
\operatorname{Coim}(\ell)=\operatorname{Coker}(\operatorname{Ker}(\ell))=\operatorname{Coker}\left(O_{O L}\right)=\left(L, 1_{L}\right)
$$

Now we claim that, if $A \stackrel{j}{\longrightarrow} \operatorname{Coker}(\ell)$ is the canonical morphism, then $(L, \ell)$ is a generalized kernel of $j$. Indeed, we know that $\ell$ is injective and $j \ell=O_{L \operatorname{Coker}(\ell)}$. Let $D \in \mathrm{Ob}(\mathcal{C})$ be arbitrary and let $\psi \in \operatorname{Ker}\left(j^{*}\right)$. Then there is a morphism $D \stackrel{\theta}{\longrightarrow} \operatorname{Ker}(j)$ in $\mathcal{C}$ so that $\psi=i \theta$, where $\operatorname{Ker}(j) \stackrel{i}{\longrightarrow} A$ is the canonical morphism. On the other hand, since $u \ell=O_{L B}$ and $\operatorname{Im}(\ell)=$ $\operatorname{Ker}(\operatorname{Coker}(\ell))=(\operatorname{Ker}(j), i)$, Proposition 4.5 of [10] gives $(\operatorname{Ker}(j), i) \leq(\operatorname{Ker}(u), \gamma)$, where $\operatorname{Ker}(u) \stackrel{\gamma}{\longrightarrow} A$ is the canonical morphism. Hence there is a morphism $\operatorname{Ker}(j) \stackrel{w}{\longrightarrow} \operatorname{Ker}(u)$ in $\mathcal{C}$ with $i=\gamma w$ and, consequently,

$$
\psi=i \theta=(\gamma w) \theta=\gamma(w \theta)
$$

Moreover, since $(\operatorname{Ker}(u), \gamma)$ and $(L, \ell)$ are equivalent, there is a morphism $\operatorname{Ker}(u) \stackrel{s}{\longrightarrow} L$ in $\mathcal{C}$ such that $\gamma=\ell s$ (because $(\operatorname{Ker}(u), \gamma) \leq(L, \ell)$ ), and therefore

$$
\psi=\gamma(w \theta)=(\ell s)(w \theta)=\ell(s(w \theta)) \in \operatorname{Im}\left(\ell^{*}\right)
$$

This proves our claim. 


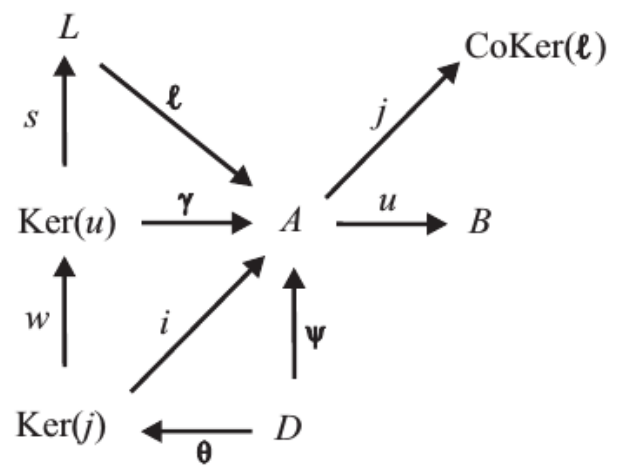

Finally, by what we have just observed, it follows that $(L, \ell)$ and $(\operatorname{Ker}(j), i)$ are equivalent, and thus we may write $\ell=i \xi$, where $\xi: L \rightarrow \operatorname{Ker}(j)$ is an isomorphism. Therefore we have the sequence of morphisms

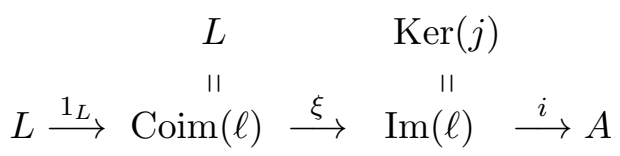

such that $i \xi 1_{L}=\ell$, which guarantees that $\bar{\ell}=\xi$ is an isomorphism.

Definition 3.5. A sequence

$$
A \stackrel{u}{\longrightarrow} B \stackrel{v}{\longrightarrow} C
$$

of morphisms in $\mathcal{C}$ is said to be exact if $(\operatorname{Im}(u), \beta)=\operatorname{Ker}(v)$, where $\beta$ is the canonical morphism.

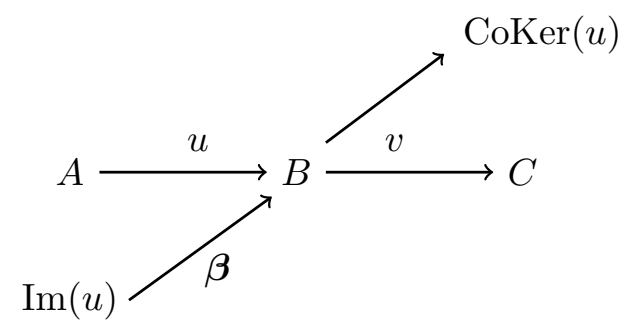

A sequence

$$
\cdots \longrightarrow A_{n} \stackrel{u_{n}}{\longrightarrow} A_{n+1} \stackrel{u_{n+1}}{\longrightarrow} A_{n+2} \longrightarrow \cdots
$$


of morphisms in $\mathcal{C}$ is said to be exact if the sequence

$$
A_{n} \stackrel{u_{n}}{\longrightarrow} A_{n+1} \stackrel{u_{n+1}}{\longrightarrow} A_{n+2}
$$

is exact for each $n$.

Now we may state the following

Theorem 3.6. For $u \in \operatorname{Mor}_{\mathcal{C}}(A, B)$ and $v \in \operatorname{Mor}_{\mathcal{C}}(B, C)$, the following conditions are equivalent:

(a) $u$ is a strict morphism and the sequence

$$
O \longrightarrow A \stackrel{u}{\longrightarrow} B \stackrel{v}{\longrightarrow} C
$$

is exact;

(b) for each $D \in \mathrm{Ob}(\mathcal{C})$, the sequence

$$
O \longrightarrow \operatorname{Mor}_{\mathcal{C}}(D, A) \stackrel{u^{*}}{\longrightarrow} \operatorname{Mor}_{\mathcal{C}}(D, B) \stackrel{v^{*}}{\longrightarrow} \operatorname{Mor}_{\mathcal{C}}(D, C)
$$

of group homomorphisms is exact, where $u^{*}(\varphi)=u \varphi$ for $\varphi \in \operatorname{Mor}_{\mathcal{C}}(D, A)$ and $v^{*}(\psi)=v \psi$ for $\psi \in \operatorname{Mor}_{\mathcal{C}}(D, B)$.

In order to prove the theorem we shall need the following auxiliary result:

Lemma 3.7. If $u \in \operatorname{Mor}_{\mathcal{C}}(A, B)$ is injective and strict, then $(A, u)$ and $(\operatorname{Im}(u), \beta)$ are equivalent.

Proof of Lemma 3.7. Let us consider the sequence

$$
A \longrightarrow \operatorname{Coim}(u) \stackrel{\bar{u}}{\longrightarrow} \operatorname{Im}(u) \stackrel{\beta}{\longrightarrow} B
$$

$A \longrightarrow \operatorname{Coim}(u)$ being the canonical morphism. By Proposition 4 of [2], $A \longrightarrow$ $\operatorname{Coim}(u)$ is an isomorphism ( $u$ is injective) and, by the strictness of $u, \bar{u}$ is an isomorphism. Thus the morphism

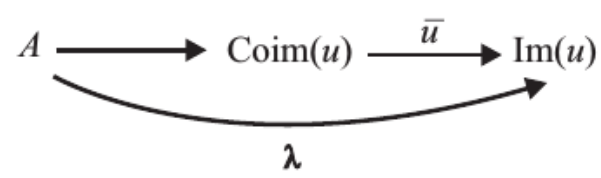

is an isomorphism. Moreover, the equality $u=\beta \lambda$ gives $(A, u) \leq(\operatorname{Im}(u), \beta)$ and the equality $\beta=u \lambda^{-1}$ gives $(\operatorname{Im}(u), \beta) \leq(A, u)$. Therefore $(A, u)$ and $(\operatorname{Im}(u), \beta)$ are equivalent, as asserted. 
Now let us turn to the

Proof of Theorem 3.6. By the definition of injectivity, we have that $u$ is injective if and only if, for each $D \in \mathrm{Ob}(\mathcal{C})$, the sequence

$$
O \longrightarrow \operatorname{Mor}_{\mathcal{C}}(D, A) \stackrel{u^{*}}{\longrightarrow} \operatorname{Mor}_{\mathcal{C}}(D, B)
$$

is exact.

$(\mathrm{a}) \Rightarrow(\mathrm{b})$ : The exactness of the sequence

$$
O \longrightarrow A \stackrel{u}{\longrightarrow} B
$$

gives

$$
\operatorname{Im}\left(O_{O A}\right)=\operatorname{Ker}\left(\operatorname{Coker}\left(O_{O A}\right)\right)=\operatorname{Ker}\left(1_{A}\right)=O=\operatorname{Ker}(u),
$$

which implies the injectivity of $u$. Thus the sequence

$$
O \longrightarrow \operatorname{Mor}_{\mathcal{C}}(D, A) \stackrel{u^{*}}{\longrightarrow} \operatorname{Mor}_{\mathcal{C}}(D, B)
$$

is exact for each $D \in \mathrm{Ob}(\mathcal{C})$. Now, let us show the exactness of the sequence

$$
\operatorname{Mor}_{\mathcal{C}}(D, A) \stackrel{u^{*}}{\longrightarrow} \operatorname{Mor}_{\mathcal{C}}(D, B) \stackrel{v^{*}}{\longrightarrow} \operatorname{Mor}_{\mathcal{C}}(D, C)
$$

where $D \in \operatorname{Ob}(\mathcal{C})$ is arbitrary. Indeed, the assumption that $(\operatorname{Im}(u), \beta)=\operatorname{Ker}(v)$ guarantees the exactness of the sequence

$$
\operatorname{Mor}_{\mathcal{C}}(D, \operatorname{Im}(u)) \stackrel{\beta^{*}}{\longrightarrow} \operatorname{Mor}_{\mathcal{C}}(D, B) \stackrel{v^{*}}{\longrightarrow} \operatorname{Mor}_{\mathcal{C}}(D, C),
$$

where $D \in \mathrm{Ob}(\mathcal{C})$ is arbitrary. If $\varphi \in \operatorname{Mor}_{\mathcal{C}}(D, A)$, then

$$
v^{*}\left(u^{*}(\varphi)\right)=v^{*}(u \varphi)=v^{*}((\beta \lambda) \varphi)=v^{*}(\beta(\lambda \varphi))=v^{*}\left(\beta^{*}(\lambda \varphi)\right)=O_{D C},
$$

$\lambda$ being as in the proof of Lemma 3.7. Hence $\operatorname{Im}\left(u^{*}\right) \subset \operatorname{Ker}\left(v^{*}\right)$. On the other hand, if $\psi \in \operatorname{Ker}\left(v^{*}\right)$, there is a $w \in \operatorname{Mor}_{\mathcal{C}}(D, \operatorname{Im}(u))$ so that

$$
\psi=\beta^{*}(w)=\beta w=\left(u \lambda^{-1}\right) w=u\left(\lambda^{-1} w\right)=u^{*}\left(\lambda^{-1} w\right)
$$

showing that $\psi \in \operatorname{Im}\left(u^{*}\right)$. Therefore $\operatorname{Ker}\left(v^{*}\right) \subset \operatorname{Im}\left(u^{*}\right)$, and $\operatorname{Im}\left(u^{*}\right)=\operatorname{Ker}\left(v^{*}\right)$.

(b) $\Rightarrow(\mathrm{a})$ : By what we have remarked at the beginning of the proof, $u$ is injective, and hence the equality $\operatorname{Ker}(u)=O=\operatorname{Im}\left(O_{O A}\right)$ guarantees the exactness of the sequence

$$
O \longrightarrow A \stackrel{u}{\longrightarrow} B
$$


Moreover, since the sequence

$$
\operatorname{Mor}_{\mathcal{C}}(D, A) \stackrel{u^{*}}{\longrightarrow} \operatorname{Mor}_{\mathcal{C}}(D, B) \stackrel{v^{*}}{\longrightarrow} \operatorname{Mor}_{\mathcal{C}}(D, C)
$$

is exact for each $D \in \mathrm{Ob}(\mathcal{C}),(A, u)$ is a generalized kernel of $v$. Thus $u$ is a strict morphism by Example 3.4. Consequently, by $\operatorname{Lemma} 3.7,(\operatorname{Im}(u), \beta)$ and $(A, u)$ are equivalent. Therefore $(\operatorname{Im}(u), \beta)=\operatorname{Ker}(v)$, which concludes the proof.

Corollary $3.8[2,5]$. If $\mathcal{C}$ is an abelian category, $u \in \operatorname{Mor}_{\mathcal{C}}(A, B)$ and $v \in$ $\operatorname{Mor}_{\mathcal{C}}(B, C)$, the following conditions are equivalent:

(a) the sequence

$$
O \longrightarrow A \stackrel{u}{\longrightarrow} B \stackrel{v}{\longrightarrow} C
$$

is exact;

(b) for each $D \in \mathrm{Ob}(\mathcal{C})$, the sequence

$$
O \longrightarrow \operatorname{Mor}_{\mathcal{C}}(D, A) \stackrel{u^{*}}{\longrightarrow} \operatorname{Mor}_{\mathcal{C}}(D, B) \stackrel{v^{*}}{\longrightarrow} \operatorname{Mor}_{\mathcal{C}}(D, C)
$$

of group homomorphisms is exact.

Proof. Follows immediately from Theorem 3.6 since, by definition, every morphism in an abelian category is strict.

Example 3.9. The condition that $u$ is strict is essential for the validity of (a) $\Rightarrow$ (b) in Theorem 3.6.

Indeed, let $\mathcal{C}$ be the semiabelian category of abelian topological groups. Let $A$ be the additive group of real numbers endowed with the discrete topology, $B$ the additive group of real numbers endowed with the usual topology, $u$ the group homomorphism from $A$ into $B$ given by $u(x)=x$, and $v$ the group homomorphism from $B$ into $B$ given by $v(x)=0$. Then $A, B \in \mathrm{Ob}(\mathcal{C}), u \in$ $\operatorname{Mor}_{\mathcal{C}}(A, B), v \in \operatorname{Mor}_{\mathcal{C}}(B, B)$ and $\bar{u}=u$ (thus $u$ is not a strict morphism). Moreover, the sequence

$$
O \longrightarrow A \stackrel{u}{\longrightarrow} B \stackrel{v}{\longrightarrow} B
$$

is obviously exact. We claim that the sequence

$$
\operatorname{Mor}_{\mathcal{C}}(B, A) \stackrel{u^{*}}{\longrightarrow} \operatorname{Mor}_{\mathcal{C}}(B, B) \stackrel{v^{*}}{\longrightarrow} \operatorname{Mor}_{\mathcal{C}}(B, B)
$$

is not exact. In fact, if it were, the fact that $1_{B} \in \operatorname{Ker}\left(v^{*}\right)$ would imply the existence of a morphism $B \stackrel{w}{\longrightarrow} A$ in $\mathcal{C}$ such that $u^{*}(w)=1_{B}$, which would 
furnish $x=1_{B}(x)=u(w(x))=w(x)$ for $x \in B$; but the mapping $x \in B \mapsto x \in$ $A$ is not continuous.

The dual exactness criterion of the one given in Theorem 3.6 reads:

Theorem 3.10. For $u \in \operatorname{Mor}_{\mathcal{C}}(A, B)$ and $v \in \operatorname{Mor}_{\mathcal{C}}(B, C)$, the following conditions are equivalent:

(a) $v$ is a strict morphism and the sequence

$$
A \stackrel{u}{\longrightarrow} B \stackrel{v}{\longrightarrow} C \longrightarrow O
$$

is exact;

(b) for each $D \in \mathrm{Ob}(\mathcal{C})$, the sequence

$$
O \longrightarrow \operatorname{Mor}_{\mathcal{C}}(C, D) \stackrel{v_{*}}{\longrightarrow} \operatorname{Mor}_{\mathcal{C}}(B, D) \stackrel{u_{*}}{\longrightarrow} \operatorname{Mor}_{\mathcal{C}}(A, D)
$$

of group homomorphisms is exact, where $v_{*}(\psi)=\psi v$ for $\psi \in \operatorname{Mor}_{\mathcal{C}}(C, D)$ and $u_{*}(\varphi)=\varphi u$ for $\varphi \in \operatorname{Mor}_{\mathcal{C}}(B, D)$.

As in Theorem 3.6, the strictness of $v$ is essential for the validity of (a) $\Rightarrow$ (b) in Theorem 3.10. Finally, as before, we may mention the following

Corollary 3.11 [2]. If $\mathcal{C}$ is an abelian category, $u \in \operatorname{Mor}_{\mathcal{C}}(A, B)$ and $v \in \operatorname{Mor}_{\mathcal{C}}(B, C)$, the following conditions are equivalent:

(a) the sequence

$$
A \stackrel{u}{\longrightarrow} B \stackrel{v}{\longrightarrow} C \longrightarrow O
$$

is exact;

(b) for each $D \in \mathrm{Ob}(\mathcal{C})$, the sequence

$$
O \longrightarrow \operatorname{Mor}_{\mathcal{C}}(C, D) \stackrel{v_{*}}{\longrightarrow} \operatorname{Mor}_{\mathcal{C}}(B, D) \stackrel{u_{*}}{\longrightarrow} \operatorname{Mor}_{\mathcal{C}}(A, D)
$$

of group homomorphisms is exact.

\section{References}

[1] M. Akkar, Espaces vectoriels bornologiques $K$-convexes, Indag. Math., 73 (1970), 82-95.

[2] A. Andreotti, Généralités sur les catégories abéliennes, Séminaire A. Grothendieck (Algèbre Homologique), Exposé 2, Faculté des Sciences de Paris (1957).

[3] N. Bourbaki, Algebra I, Chapters 1-3, Second printing, Springer-Verlag, Berlin, Heidelberg, New York (1989). 
[4] P. Freyd, Abelian Categories, Harper and Row, New York (1964); republished in: Reprints in Theory and Applications of Categories, 3 (2003), 23-164.

[5] A. Grothendieck, Sur quelques points d'algèbre homologique, Thoku Math. J., 9 (1957), 119-221.

[6] H. Hogbe-Nlend, Bornologies and Functional Analysis, Notas de Matemática 62, NorthHolland, Amsterdam (1977).

[7] G. Köthe, Topological Vector Spaces I, Grundlehren der mathematischen Wissenschaften 159, Springer-Verlag, Berlin, Heidelberg, New York (1969).

[8] V.P. Palamodov, Homological methods in the theory of locally convex spaces, Russian Math. Surveys, 26 (1971), 1-64.

[9] D.P. Pombo Jr., Module bornologies, Comment. Math. (Prace Mat.), 36 (1996), 179-188.

[10] D.P. Pombo Jr., On semiabelian categories in Functional Analysis and Topological Algebra, arXiv: 1409.4348v2 [math. FA] (2015).

[11] A.P. Robertson \& W. Robertson, Topological Vector Spaces, Second edition, Cambridge University Press, Cambridge (1973).

[12] P. Schneider, Nonarchimedean Functional Analysis, Springer Monographs in Mathematics, Springer-Verlag, Berlin, Heidelberg, New York (2002).

[13] S. Warner, Topological Fields, Notas de Matemática 126, North-Holland, Amsterdam (1989). 